(6) OPEN ACCESS

\section{- Additional material is published online only. To view please visit the journal online (http://dx.doi.org/10.1136/ archdischild-2013-305708). \\ ${ }^{1}$ School of Information Technology and Electrical Engineering, The University of Queensland, Brisbane, Queensland, Australia ${ }^{2}$ Department of Respiratory and Sleep Medicine, The Mater Children's Hospital, South Brisbane, Queensland, Australia \\ ${ }^{3}$ Australasian Paediatric Endocrine Group, Mater Medical Research Institute, Brisbane, Queensland Australia}

\section{Correspondence to} Dr Philip Terrill, School of Information Technology and Electrical Engineering, The University of Queensland, Brisbane, QLD 4072, Australia; p.terrill@uq.edu.au

Received 21 November 2013 Revised 23 June 2014 Accepted 25 June 2014 Published Online First 25 July 2014

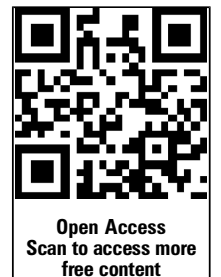

\title{
Nocturnal oxygen saturation profiles of healthy term infants
}

\author{
Philip Ian Terrill, ${ }^{1}$ Carolyn Dakin, ${ }^{2}$ Ian Hughes, ${ }^{3}$ Maggie Yuill, ${ }^{2}$ Chloe Parsley $^{2}$
}

ABSTRACT
Objective Pulse oximetry is used extensively in hospital and home settings to measure arterial oxygen saturation $\left(\mathrm{SpO}_{2}\right)$. Interpretation of the trend and range of $\mathrm{SpO}_{2}$ values observed in infants is currently limited by a lack of reference ranges using current devices, and may be augmented by development of cumulative frequency (CF) reference-curves. This study aims to provide reference oxygen saturation values from a prospective longitudinal cohort of healthy infants.

Design Prospective longitudinal cohort study.

Setting Sleep-laboratory.

Patients 34 healthy term infants were enrolled, and studied at 2 weeks, 3, 6, 12 and 24 months of age $(\mathrm{N}=30,25,27,26,20$, respectively).

Interventions Full overnight polysomnography, including $2 \mathrm{~s}$ averaging pulse oximetry (Masimo Radical). Main outcome measurements Summary $\mathrm{SpO}_{2}$ statistics (mean, median, 5th and 10th percentiles) and $\mathrm{SpO}_{2} \mathrm{CF}$ plots were calculated for each recording. CF reference-curves were then generated for each study age. Analyses were repeated with sleep-state stratifications and inclusion of manual artefact removal.

Results Median nocturnal $\mathrm{SpO}_{2}$ values ranged between $98 \%$ and $99 \%$ over the first 2 years of life and the CF reference-curves shift right by $1 \%$ between 2 weeks and 3 months. CF reference-curves did not change with manual artefact removal during sleep and did not vary between rapid eye movement (REM) and non-REM sleep. Manual artefact removal did significantly change summary statistics and CF reference-curves during wake. Conclusions $\mathrm{SpO}_{2} \mathrm{CF}$ curves provide an intuitive visual tool for evaluating whether an individual's nocturnal $\mathrm{SpO}_{2}$ distribution falls within the range of healthy agematched infants, thereby complementing summary statistics in the interpretation of extended oximetry recordings in infants.

\section{INTRODUCTION}

Modern pulse-oximeters non-invasively measure and record high temporal resolution arterial blood haemoglobin oxygen saturations $\left(\mathrm{SpO}_{2}\right)$, making them a valuable clinical tool in a range of paediatric clinical applications. Published reference ranges have documented the normal ranges of summary measurements such as baseline $\mathrm{SpO}_{2}$, mean $\mathrm{SpO}_{2}$ and median $\mathrm{SpO}_{2}$ values, ${ }^{1-9}$ with more recent studies expanding reference statistics to include ranges, nadirs, 5 th and 10 th percentiles of $\mathrm{SpO}_{2}$ distributions and time below specific $\mathrm{SpO}_{2}$ levels. ${ }^{10} 11$ However, many of these studies used devices which are no longer available in clinical practice or present selected parameters specific to particular models and averaging time settings. Furthermore, with

\section{What is already known on this topic}

- Extended recording of pulse oximetry can provide important information about the health of an individual relatively non-invasively in a hospital environment.

- $\mathrm{SpO}_{2}$ reference data, stratified by sleep-state in term infants aged 2 weeks-24 months, are currently not available for modern high temporal resolution pulse-oximeters.

- Summary statistics, such as mean and medians, do not capture the overall distribution of extended $\mathrm{SpO}_{2}$ recordings.

\section{What this study adds}

- This study provides reference $\mathrm{SpO}_{2}$ statistics, stratified by sleep-state, for healthy term infants aged 2 weeks-2 years, using a current generation pulse-oximeter.

- Cumulative frequency reference-curves provide an intuitive visual tool for rapidly evaluating whether an individual's $\mathrm{SpO}_{2}$ distribution is within a healthy range.

- Cumulative frequency plots complement summary statistics and visual inspection of raw data in the clinical interpretation of nocturnal $\mathrm{SpO}_{2}$ recordings.

modern oximeters capable of recording individual data points for extended periods, it is highly desirable to develop normative measurements which quantify the overall $\mathrm{SpO}_{2}$ distribution. This would be of particular value in the titration of supplemental oxygen requirements in infants with chronic neonatal lung disease $\mathrm{e}^{12-15}$ and screening for obstructive sleep apnoea. ${ }^{16}$

One method of presenting $\mathrm{SpO}_{2}$ data in a graphical, and therefore perhaps more readily interpretable manner, is the $\mathrm{SpO}_{2}$ cumulative frequency (CF) curve. These are produced by some commercial oximetry software, and reference values for healthy preterm infants have been presented by Harigopal et $a l^{17}$ and $\mathrm{Ng}$ et al. ${ }^{18}$ In this approach, the $\mathrm{SpO}_{2} \mathrm{CF}$ curve was generated for each infant by calculating the proportion of the recording spent at or below each discrete $\mathrm{SpO}_{2}$ saturation level. CF reference-curves were then generated by calculating the 5 th and 50 th centiles at each $\mathrm{SpO}_{2}$ value for the study population. Thus, 
analogous to using a growth chart, by plotting the $\mathrm{SpO}_{2} \mathrm{CF}$ curve for a subject of interest over the reference-curves, it can be easily observed whether the infant's $\mathrm{SpO}_{2}$ profile falls within the range expected for a healthy infant. This study thus aimed to present reference ranges for $\mathrm{SpO}_{2}$ summary statistics and CF reference-curves for healthy term infants from the neonatal period to 2 years of age. The impact of manual artefact removal and sleep-state stratification on CF reference-curves and summary statistics are also investigated.

\section{METHODS}

\section{Subjects and procedures}

Complete details of the protocol have been described elsewhere. ${ }^{19-22}$ Recruitment was from antenatal clinics during the third trimester of an uneventful singleton pregnancy, with 34 healthy Caucasian infants (16 girls) enrolled following birth.
The intention of the initial study design was to collect normative data on various cardiorespiratory parameters, including oxygen saturation. The sample size was selected to ensure statistical power to detect maturational changes in key summary variables such as number of $\mathrm{SpO}_{2}$ desaturation events, while being a feasible number in which detailed sleep studies could be performed. Infants were eligible if they were: born by normal vaginal delivery or planned caesarean section at term $(38-42$ weeks) with normal birth weight (10th-90th percentile), ${ }^{23}$ Apgar score $>7$ at $5 \mathrm{~min}$ and were from a non-smoking household. Exclusion criteria included congenital or anatomical abnormality; sudden infant death syndrome sibling; supplemental oxygen requirement $>5$ min following delivery; or a strong maternal family history of atopy.

This prospective cohort was studied with full overnight polysomnogram within the Queensland Paediatric Sleep Service at the

Table 1 Participant characteristics and $\mathrm{SpO}_{2}$ summary statistics calculated without manual artefact removal

\begin{tabular}{|c|c|c|c|c|c|c|c|c|}
\hline \multirow{2}{*}{$\begin{array}{l}\text { Summary statistic } \\
2 \text { weeks ( } N=30,13 \text { female) }\end{array}$} & \multicolumn{2}{|l|}{ All } & \multicolumn{2}{|c|}{ non-REM/QS } & \multicolumn{2}{|l|}{ REM/AS } & \multicolumn{2}{|l|}{ Wake } \\
\hline & & & & & & & & \\
\hline Age (days) & $14 \pm 3$ & & & & & & & \\
\hline Duration (min) & 549.6 & (396-1024) & 153.9 & $(114-544)$ & 203.8 & $(93-270)$ & 156.0 & $(38-357)$ \\
\hline Artefact-free duration (min) & 503.0 & $(343-635)$ & 151.2 & $(113-220)$ & 193.9 & (93-266) & 117.6 & $(15-231)$ \\
\hline SAT5 (\%Hb) & 95.0 & (88-97) & 96.0 & $(85-98)$ & 95.0 & (88-97) & 92.0 & (73-99) \\
\hline SAT10 (\%Hb) & 96.0 & (92-98) & 97.0 & $(93-98)$ & 96.0 & (92-98) & 96.0 & (77-99) \\
\hline Median $\mathrm{SpO}_{2}(\% \mathrm{Hb})$ & 98.0 & $(95-100)$ & 98.0 & $(95-100)$ & 97.5 & $(95-100)$ & 98.0 & $(96-100)$ \\
\hline Mean $\mathrm{SpO}_{2}(\% \mathrm{Hb})$ & 97.6 & (95-99) & 97.9 & $(95-99)$ & 97.2 & (95-99) & 97.6 & $(93-100)$ \\
\hline \multicolumn{9}{|l|}{3 months ( $N=25,10$ female) } \\
\hline Age (days) & $97 \pm 9$ & & & & & & & \\
\hline Duration (min) & 534.5 & $(411-660)$ & 249.5 & $(151-327)$ & 189.5 & $(86-302)$ & 75.5 & $(8-313)$ \\
\hline Artefact-free duration (min) & 492.9 & $(391-621)$ & 249.5 & $(149-327)$ & 189.5 & $(73-302)$ & 60.6 & $(8-169)$ \\
\hline SAT5 (\%Hb) & 97.0 & (90-99) & 98.0 & $(94-99)$ & 97.0 & $(93-99)$ & 85.0 & $(68-99)$ \\
\hline SAT10 (\%Hb) & 98.0 & (95-99) & 98.0 & $(95-100)$ & 98.0 & (94-99) & 91.0 & $(76-100)$ \\
\hline Median $\mathrm{SpO}_{2}(\% \mathrm{Hb})$ & 99.0 & $(97-100)$ & 99.0 & $(97-100)$ & 99.0 & $(97-100)$ & 99.0 & $(97-100)$ \\
\hline Mean $\mathrm{SpO}_{2}(\% \mathrm{Hb})$ & 98.5 & $(97-100)$ & 98.7 & $(97-100)$ & 98.9 & $(97-100)$ & 97.3 & $(92-100)$ \\
\hline \multicolumn{9}{|l|}{6 months ( $N=27,10$ female) } \\
\hline Age (days) & $198 \pm 6$ & & & & & & & \\
\hline Duration (min) & 498.6 & $(367-680)$ & 270.6 & $(160-421)$ & 147.0 & $(88-229)$ & 91.5 & $(20-214)$ \\
\hline Artefact-free duration (min) & 462.5 & (351-654) & 262.0 & $(160-397)$ & 140.0 & $(83-227)$ & 66.7 & $(14-179)$ \\
\hline SAT5 (\%Hb) & 97.0 & $(95-98)$ & 97.0 & $(95-99)$ & 97.0 & $(95-99)$ & 96.0 & $(83-98)$ \\
\hline SAT10 (\%Hb) & 97.0 & (96-99) & 98.0 & $(96-99)$ & 98.0 & (96-99) & 97.0 & $(86-100)$ \\
\hline Median $\mathrm{SpO}_{2}(\% \mathrm{Hb})$ & 98.0 & $(97-100)$ & 98.0 & $(97-100)$ & 99.0 & $(97-100)$ & 99.0 & $(98-100)$ \\
\hline Mean $\mathrm{SpO}_{2}(\% \mathrm{Hb})$ & 98.3 & $(97-100)$ & 98.3 & $(97-100)$ & 98.5 & $(97-100)$ & 98.2 & $(96-99)$ \\
\hline \multicolumn{9}{|l|}{12 months ( $\mathrm{N}=26,10$ female) } \\
\hline Age (days) & $371 \pm 14$ & & & & & & & \\
\hline Duration (min) & 499.0 & (259-744) & 272.3 & $(131-532)$ & 128.3 & $(47-216)$ & 72.0 & $(3-251)$ \\
\hline Artefact-free duration (min) & 442.8 & $(205-612)$ & 267.1 & $(119-355)$ & 121.9 & $(47-216)$ & 43.4 & $(3-132)$ \\
\hline SAT5 (\%Hb) & 97.0 & (89-99) & 97.0 & $(95-99)$ & 97.0 & (83-99) & 95.5 & $(69-99)$ \\
\hline SAT10 (\%Hb) & 98.0 & (95-99) & 98.0 & $(95-99)$ & 98.0 & (94-99) & 97.0 & (75-99) \\
\hline Median $\mathrm{SpO}_{2}(\% \mathrm{Hb})$ & 99.0 & $(96-100)$ & 98.5 & $(96-100)$ & 99.0 & $(96-100)$ & 99.0 & $(97-100)$ \\
\hline Mean $\mathrm{SpO}_{2}(\% \mathrm{Hb})$ & 98.4 & $(96-100)$ & 98.4 & $(96-100)$ & 98.5 & $(96-100)$ & 98.2 & $(95-100)$ \\
\hline \multicolumn{9}{|l|}{24 months ( $N=20,5$ female) } \\
\hline Age (days) & $725 \pm 8$ & & & & & & & \\
\hline Duration (min) & 486.3 & (323-658) & 337.8 & $(240-512)$ & 112.8 & $(41-188)$ & 37.5 & $(0-155)$ \\
\hline Artefact-free duration (min) & 481.5 & $(220-582)$ & 306.7 & $(181-385)$ & 103.9 & (38-188) & 28.9 & $(0-138)$ \\
\hline SAT5 (\%Hb) & 97.5 & (96-99) & 98.0 & $(96-99)$ & 98.0 & (95-99) & 97.0 & (73-98) \\
\hline SAT10 $(\% \mathrm{Hb})$ & 98.0 & (96-99) & 98.0 & $(96-100)$ & 98.0 & (96-99) & 97.0 & $(75-100)$ \\
\hline Median $\mathrm{SpO}_{2}(\% \mathrm{Hb})$ & 99.0 & $(97-100)$ & 99.0 & $(97-100)$ & 99.0 & $(97-100)$ & 99.0 & $(97-100)$ \\
\hline Mean $\mathrm{SpO}_{2}(\% \mathrm{Hb})$ & 98.7 & $(97-100)$ & 98.7 & $(97-100)$ & 98.8 & $(97-100)$ & 97.9 & $(94-100)$ \\
\hline
\end{tabular}

All summary statistics are calculated from $\mathrm{SpO}_{2}$ data without manual artefact removal. Data are presented for the entire recording (All), and stratified according to sleep-states non-REM, REM and wake for children at 3 months and older; and quiet sleep (QS), active sleep (AS) and wake for children at the neonatal study. SAT5, 5 th percentile SpO 2 value; $\mathrm{SAT} 10,10$ th percentile $\mathrm{SpO}_{2}$ value. Values are presented as median (range) or mean $\pm \mathrm{SD}$. REM, rapid eye movement. 
Mater Children's Hospital in the neonatal period (day 5-20) and then at 3, 6, 12 and 24 months of age. Scheduled studies were conducted if the infant was well and free of inter-current illness (on physician examination). In the event of a viral respiratory illness, studies were rescheduled to at least 2 weeks after clinical resolution of the illness. A polysomnographic sleep-study was conducted using an EMBLA acquisition system (Embla N7000 system, Natus Medical Inc) and Somnologica software (Somnologica V.3.3.2 Build 1559). As part of this montage, arterial oxygen saturation was measured using a Masimo Radical (software-build 4.1, Masimo Corporation, Irvine, California, USA) pulse-oximeter with $2 \mathrm{~s}$ averaging and $1 \mathrm{~Hz}$ temporal resolution. All procedures were completed between March 2006 and July 2009, and were compliant with the Thoracic Society of Australia and New Zealand recommendations for paediatric sleep laboratories. ${ }^{24}$

Each study was manually scored by a trained, experienced scorer, with sleep-staging conducted using Anders criteria at the neonatal study $^{25}$ and the AASM manual for scoring sleep for infants aged 3 months and over, ${ }^{26}$ as recommended by Grigg-Damberger et al. $^{27}$ This study was approved by the Mater Health Services Human Research Ethics Committee (Number 952C).

\section{Data analysis}

The nocturnal $\mathrm{SpO}_{2}$ recording for each infant, at each of the study ages, was exported from Somnologica into the European Data Format (.edf) and then imported into MATLAB (Mathworks, Natick, Massachusetts, USA) for further analysis. To account for small analogue acquisition calibration errors
$(<0.5 \%)$ when digitally sampled at the polysomnogram system, $\mathrm{SpO}_{2}$ values were rounded to the nearest integer percentage value. The Masimo Radical oximeter has sophisticated artefact detection algorithms which mark artefactual periods by setting $\mathrm{SpO}_{2}=0 \%$ and, consequently, samples of data less than $25 \%$ after rounding were removed from further analysis.

The $\mathrm{SpO}_{2}$ cumulative distribution function (CDF) describes the proportion of the recording spent at or below each integer $\mathrm{SpO}_{2}$ value, that is, for a recording of $\mathrm{SpO}_{2}$ samples $\mathrm{S}$, and integer $\mathrm{SpO}_{2}$ values (x):

$$
\mathrm{CF}(\mathrm{x})=\mathrm{P}(\mathrm{S} \leq \mathrm{x})
$$

For each infant, at each of the five study points, a CF curve was generated using the method described by Harigopal et al. ${ }^{17}$ Reference CF reference-curves were then generated by identifying the minimum, 25th percentile, median, 75th percentile and maximum value across the study population at each discrete $\mathrm{SpO}_{2}$ value to generate $\mathrm{CF}$ reference-curves representing the expected range of values for healthy infants at the specified age. In addition, the mean, median, 5th and 10th percentiles of $\mathrm{SpO}_{2}$ values (SAT5 and SAT10) were calculated for each study.

Secondary analysis included the stratification of results according to the sleep-states of wake, non-rapid eye movement (REM) and REM (wake, quiet sleep and active sleep for infants at 2 weeks); . $^{29}$ the manual identification and removal of movement artefacts in the $\mathrm{SpO}_{2}$ channel (defined as changes in $\mathrm{SpO}_{2}$ corresponding to movement noted on video recording)
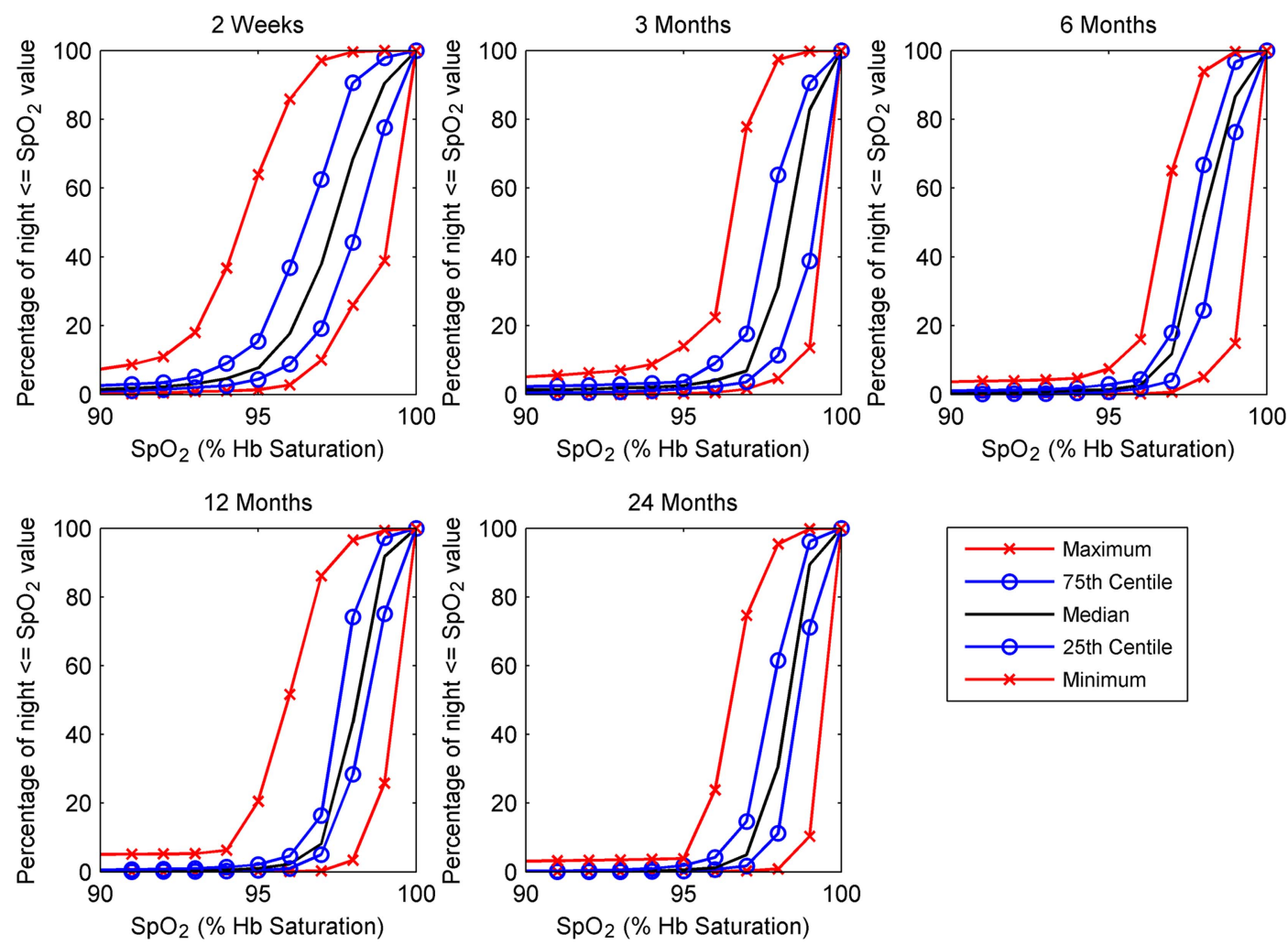

Figure 1 Cumulative frequency (CF) reference-curves of $\mathrm{SpO}_{2}$ profiles for a population of healthy term infants aged 2 weeks, 3, 6, 12 and 24 months. CF reference-curves are derived from the nocturnal $\mathrm{SpO}_{2}$ recordings of 30,25, 27, 26 and 20 infants at 2 weeks, 3, 6, 12 and 24 months of age, respectively, in a longitudinal follow-up study. Results are presented for the whole nocturnal recording, using CF plots calculated from oximetry data without manual artefact removal. The CF plot for an individual shows the percentage of a recording spent at, or below a particular $\mathrm{SpO}_{2}$ value, while the CF reference-curve shows the range of percentage time spent below a particular $\mathrm{SpO}_{2}$ value in the healthy term population. For example, it may be seen that in healthy infants at 2 weeks of age, between $0 \%$ and $60 \%$ of a recording may be spent with an $\mathrm{SpO}_{2}$ of $95 \%$ or less. Notably, the CF reference-curves shift right by $1 \%-2 \%$ between 2 weeks and 3 months, where they remain stable up to 24 months. 
which were not automatically detected; and comparison of CF reference-curves to those previously presented for healthy preterm children. ${ }^{17}$ Wilcoxon-Sign-Rank tests were used to compare summary statistics in sleep-state, age and artefact rejection comparisons. $\mathrm{p}$ Value $<0.01$ was considered statistically significant. In the event of a missing study for an infant at a particular age, that infant was excluded from statistical tests in relevant age comparisons.

\section{RESULTS}

Of the 34 children enrolled, 31, 25, 27, 26 and 20 participants completed studies at 2 weeks, 3, 6, 12 and 24 months, respectively (see online data supplement 1.1, including figure S1). One study at 2 weeks had less than 60 min of artefact-free $\mathrm{SpO}_{2}$ data and was removed from analysis. Summary statistics of the $\mathrm{SpO}_{2}$ profiles calculated without manual artefact rejection for the infant population are presented in table 1 . As expected, mean duration and percentage of study time of REM decreased with maturation, while non-REM percentage of study time increased. SAT5 was lower during wake than non-REM and REM at 2 weeks and 3 months $(p<0.01)$ and SAT10 for the same comparison at 3 months $(p<0.01)$. There was an increase in median values of SAT5 and SAT10 between 2 weeks and 3 months of age for pooled non-REM and REM stratifications $(p<0.01)$. SAT5 and SAT10 increased during wake between 3 and 6 months $(p<0.01)$. The Masimo Radical pulse-oximeter was generally effective in identifying artefact $(82.5 \%, 66.3 \%, 80.1 \%, 93.3 \%$ and $90.0 \%$ of total manually scored artefacts in infants at 2 weeks, 3, 6, 12 and
24 months, respectively). Complete summary statistics for analysis with manual artefact rejection are presented in the online data supplement 1.2. Analysis with manual artefact rejection did not change the population median by $>2 \%$ for any summary statistics for pooled sleep-states or in REM or non-REM, but during wake did result in a statistically significant increase of median SAT5 at 2 weeks, 3 and 12 months $(\mathrm{p}<0.01)$ and a notable increase in the lower bound of the range for SAT5 and SAT10 (up to 23\%).

Figure 1 shows the population minimum, 25th, 50th and 75 th percentiles and maximum $\mathrm{SpO}_{2} \mathrm{CF}$ reference-curves generated for the study population at each of 2 weeks, 3, 6, 12 and 24 months of age. Plots are shown for the full nocturnal recording without manual artefact removal. The CF reference-curves shift right by $1 \%$ between 2 weeks and 3 months, where they remain stable up to 24 months. The same CF reference-curves overlaid with reference-curves generated using manual artefact removal are presented in figure 2. There is no qualitative change to median or minimum population curves, but the maximum curve shows a reduced percentage of the night spent below $94 \%$ saturation. Tabulated data for these $\mathrm{SpO}_{2} \mathrm{CDF}$ nomograms and those generated with sleep-state stratifications are presented in online data supplement 1.3 , while commonly used summary statistics derived from these (percentage of night with $\mathrm{SpO}_{2}$ below $92 \%$ and $95 \%$ ) are tabulated in online data supplement 1.4 .

Figure 3 compares the CF reference-curves presented in figure 1 with those presented in literature for healthy preterm infants at the same corrected age of 2 weeks post-term. ${ }^{17}$ The CF reference-curves for the preterm infant group are shifted to
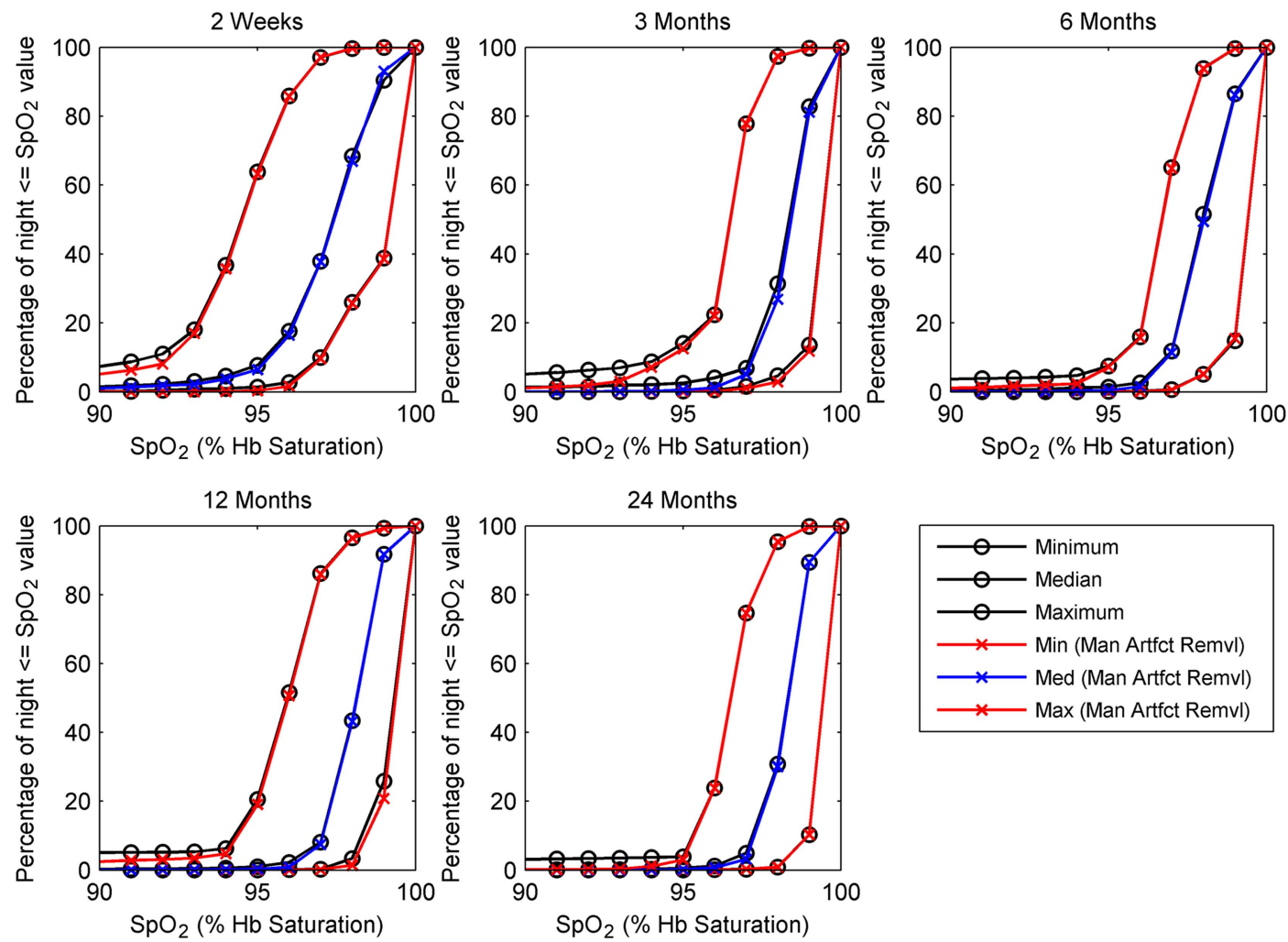

Figure $2 \mathrm{SpO}_{2}$ cumulative frequency (CF) reference-curves for healthy infants aged 2 weeks-24 months, generated with and without manual artefact removal. CF reference-curves are shown for $\mathrm{SpO}_{2}$ data from the whole nocturnal recording. At $\mathrm{SpO}_{2}$ values greater than $95 \%$, there is no discernible deviation between nomogram curves generated with and without manual artefact removal. At lower $\mathrm{Spo}_{2}$ values, the maximum curve for data without manual artefact removal remains higher than the respective nomogram curve with manual artefact removal, indicating that artefact manually removed tends to be at lower $\mathrm{SpO}_{2}$ values. Max (Man Artfct Remvl), population maximum reference-curve generated with manual artefact removal; Med (Man Artfct Remvl), population median reference-curve generated with manual artefact removal; Min (Man Artfct Remvl), population minimum reference-curve generated with manual artefact removal. 


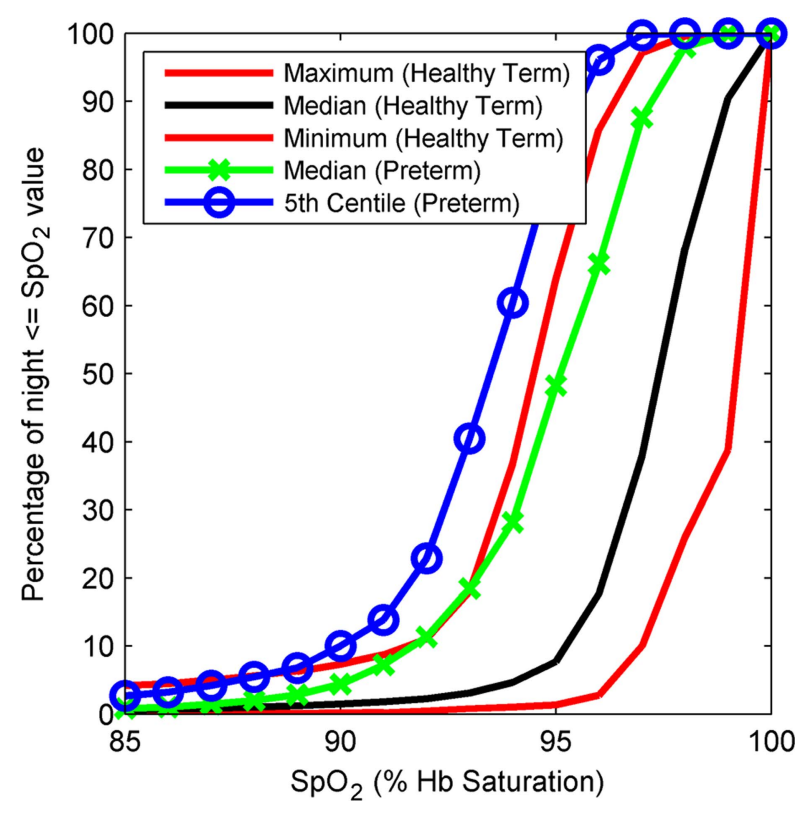

Figure $3 \quad \mathrm{SpO}_{2}$ cumulative frequency (CF) reference-curves for healthy term infants and healthy preterm infants. CF reference-curves for healthy infants at 2 weeks, overlaid with the reference-curves presented by Harigopal et $a l^{17}$ for healthy preterm infants (median gestational age at birth of 33 weeks; median age at study of 2 weeks). The reference-curves for the preterm infant group are shifted to the left relative to the term infant group (median curve shifted approximately $3 \%-4 \% \mathrm{SpO}_{2}$ ). Physiological differences in the oxy-haemoglobin dissociation curve, use of different pulse-oximeter models and the extent of manual artefact removal may account for these differences.

the left relative to the healthy infant group (median curve shifted approximately 3\%-4\% $\mathrm{SpO}_{2}$ ).

\section{DISCUSSION}

The objective of this study was to present reference ranges of nocturnal $\mathrm{SpO}_{2}$ recordings from a prospective cohort of healthy term infants at 2 weeks, 3, 6, 12 and 24 months of age using both conventional summary statistics and $\mathrm{CF}$ reference-curves. To our knowledge, this is the first study to present reference ranges recorded using a Masimo Radical pulse-oximeter with $2 \mathrm{~s}$ averaging in a prospective cohort of healthy term infants from 2 weeks to 24 months. This study was conducted in a sleep-laboratory and, as such, secondary analyses investigated the impact of sleep-state and manual artefact identification on reference ranges.

The $\mathrm{SpO}_{2}$ summary statistics presented for entire nocturnal recordings are generally consistent with the literature despite the recognised variations in recorded $\mathrm{SpO}_{2}$ values between different oximeter makes and models. ${ }^{30}$ In particular, the population range of mean and median nocturnal $\mathrm{SpO}_{2}$ values was between $95 \%$ and $100 \%$ over the first 2 years. ${ }^{2} 563132$ Median SAT5 was $95 \%$ at 2 weeks which supports existing studies that show healthy term neonates have nocturnal SAT5 values as low as $95 \%{ }^{10}$ and these lower end $\mathrm{SpO}_{2}$ values tend to increase and stabilise over the first few months of life. ${ }^{31}$ These results provide important normative data using a current generation high temporal resolution pulse-oximeter. While this is the first study to present CF reference-curves for healthy term infants, these curves have previously been presented in literature for a cohort of healthy preterm infants. ${ }^{17}$ It was noted in the results that there was a left shift of $3 \%-4 \%$ in the median curve for preterm infants indicating that $\mathrm{SpO}_{2}$ values are lower in the preterm group. Physiological differences in the oxy-haemoglobin dissociation curve, use of different pulse-oximeter models ${ }^{30}$ and the extent of artefact removal may account for these differences.

Sleep-state stratification confirmed earlier work that there is no clinically significant difference $(>2 \%)$ in summary measures of $\mathrm{SpO}_{2}$ between REM and non-REM sleep-states, ${ }^{1}$ and this was reflected in $\mathrm{CF}$ reference-curves (see online data supplement 1.3). Although we found that SAT5 and SAT10 were lower during wake than sleep at 2 weeks and 3 months, this may be related to the additional likelihood of movement artefact during wake which was not detected by the automatic algorithm. While results indicated that Masimo Radical's artefact identification was good, manual artefact removal during wakefulness resulted in an increase in summary statistics (particularly SAT5) and deviation in CDF's (observed as lower percentage of time spent below 90\%). No difference was found in either $\mathrm{SpO}_{2}$ summary statistics or $\mathrm{SpO}_{2} \mathrm{CDF}$ nomograms for pooled data, REM or non-REM stratifications following manual artefact rejection. These results suggest that clinical utility would not be limited by the inability to classify sleep-stage, although there would be benefit to manually excluding obvious movement artefacts during wake from analysis (see online data supplement 1.5 for further discussion).

The CF reference-curve has previously been proposed as a useful clinical tool for interpreting extended $\mathrm{SpO}_{2}$ recordings due to its ability to present information about the $\mathrm{SpO}_{2}$ distribution in an intuitive graphical manner. ${ }^{17}{ }^{18}$ Indeed, $\mathrm{SpO}_{2} \mathrm{CF}$ curves for individuals can be produced with some commercial oximetry and polysomnogram software, and may be readily generated using standard spreadsheet software (see online data supplement 2 provides a brief tutorial). Yet, to date, CF reference-curves for healthy term infants have been lacking. This study thus provides important reference data to allow this method to be translated into wider clinical practice. To illustrate the potential clinical utility of CF reference-curves, figure 4 displays the CF curves for an infant with chronic neonatal lung disease at 3 months gestational corrected age during an oxygen titration study superimposed on the CF reference-curves for infants at 3 months. It can be observed that when the infant is in room air, the $\mathrm{CF}$ is shifted to the left of the normative range indicating that $\mathrm{SpO}_{2}$ values are lower than may be expected in healthy infants. However, when the same infant is placed in supplemental oxygen at $0.25 \mathrm{~L} / \mathrm{min}$, the $\mathrm{CF}$ curve is within the healthy range, indicating normal $\mathrm{SpO}_{2}$ distribution. The $\mathrm{CF}$ curve alone does not discriminate between a large number of short desaturations or a smaller number of longer desaturations and, as such, best clinical utility would be achieved by using CF curves to complement summary statistics and visual inspection of large time-scale trend graphs. As with all diagnostic tests, results need to be interpreted in the wider clinical context.

There are a number of limitations in this study. First, participants were continuously supervised by an experienced sleep technologist to ensure that all sensors remained correctly attached, likely resulting in fewer artefacts than expected in a general clinical environment. While this provides the scientific advantage of improved data quality, it may limit generalisability to other clinical environments. Second, generalisability is also potentially restricted to the pulse-oximeter used in this study. However, it is a commercially available model and is commonly used in hospital settings. Finally, this was a resource-intensive sleep-laboratory based prospective cohort follow-up study, and consequently results may also be limited by a relatively small sample size. This may have particular impact on maximum and minimum CF reference-curves, and these should be interpreted as the maximum and minimum in this sample, rather than the expected limits for the wider population. Future work should 


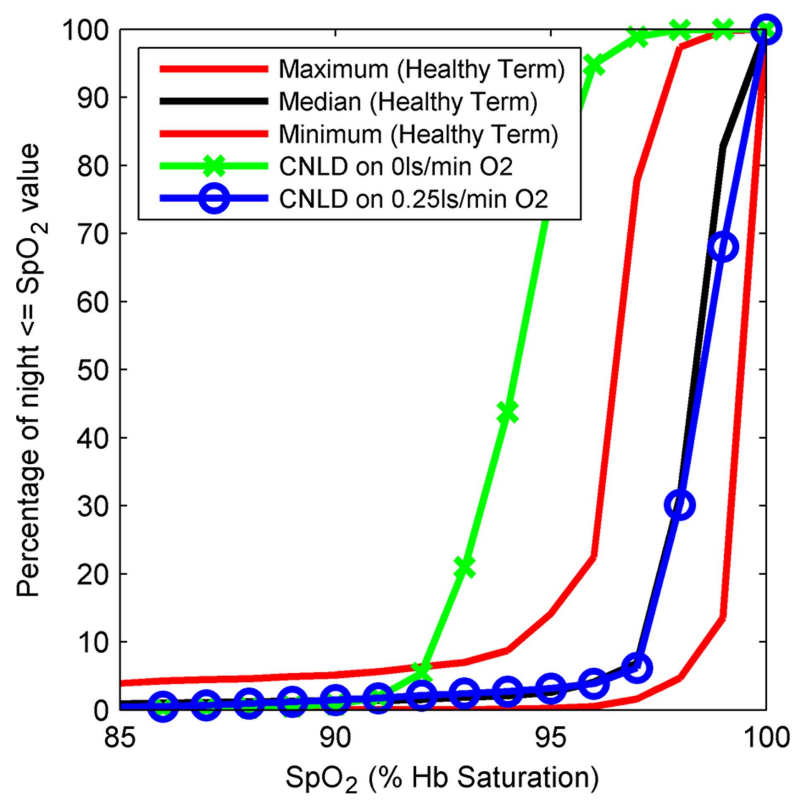

Figure 4 Effect of supplemental oxygen on an infant with chronic neonatal lung disease at 3 months corrected age during an oxygen titration study. Cumulative frequency (CF) plots for an infant with chronic neonatal lung disease at 3 months gestational corrected age, plotted over the $\mathrm{SpO}_{2}$ CF reference-curves for healthy infants at 3 months. It can be observed that when the infant is in room air, the CF plot is shifted to the left of the normative range indicating that $\mathrm{SpO}_{2}$ values are lower than may be expected in healthy infants. However, when treated with $0.25 \mathrm{~L} /$ min of supplemental oxygen, the $\mathrm{CF}$ plot is within the healthy range indicating normal $\mathrm{SpO}_{2}$ distribution.

expand these results by studying a larger sample in a more generalised clinical environment.

In conclusion, this paper presents reference ranges for healthy term infants aged from 2 weeks to 24 months of nocturnal $\mathrm{SpO}_{2}$ recordings using a Masimo Radical pulse-oximeter with $2 \mathrm{~s}$ averaging. The $\mathrm{SpO}_{2} \mathrm{CF}$ reference-curves provide an intuitive visual tool for evaluating whether an individual infant's nocturnal $\mathrm{SpO}_{2}$ distribution falls within the range of healthy age-matched infants. This reference range is potentially applicable to extended oximetry studies conducted in a range of generalised care settings and may be a useful tool in monitoring and setting appropriate supplemental oxygen levels for infants with respiratory and other diseases.

Acknowledgements The authors would like to thank the children and the families who participated in this study.

Contributors Conception and design: PIT, CD; Analysis and interpretation: PIT, CD, $I H, M Y, C P$; Drafting the manuscript for important intellectual content: PIT, $C D, I H$.

Funding This research was funded by the Mater Children's Hospital Golden Casket Research Fund.

\section{Competing interests None.}

Ethics approval The Mater Hospital Human Research Ethics Committee.

Patient consent Written, informed consent was provided by the parent/guardian of the children participating in this study.

Provenance and peer review Not commissioned; externally peer reviewed.

Data sharing statement The $\mathrm{SpO}_{2}$ cumulative frequency reference-curves presented in the manuscript are available in tabulated form in the online data supplement.

Open Access This is an Open Access article distributed in accordance with the Creative Commons Attribution Non Commercial (CC BY-NC 3.0) license, which permits others to distribute, remix, adapt, build upon this work non-commercially, and license their derivative works on different terms, provided the original work is properly cited and the use is non-commercial. See: http://creativecommons.org/ licenses/by-nc/3.0/

\section{REFERENCES}

1 Horemuzova E, Katz-Salamon M, Milerad J. Breathing patterns, oxygen and carbondioxide levels in sleeping healthy infants during the first nine months after birth. Acta Paediatr 2000;89:1284-9.

2 Hunt CE, Corwin MJ, Lister G, et al. Longitudinal assessment of hemoglobin oxygen saturation in healthy infants during the first 6 months of age. J Pediatr 1999;135:580-6.

3 Poets CF, Stebbens VA, Alexander JR, et al. Arterial oxygen saturation in preterm infants at discharge from hospital and six weeks later. J Pediatr 1992;120:447-54.

4 Poets CF, Stebbens VA, Lang JA, et al. Arterial oxygen saturation in healthy term neonates. Eur J Pediatr 1996:155:219-23.

5 Poets CF, Stebbens VA, Southall DP. Arterial oxygen saturation and breathing movements during the first year of life. J Dev Physiol 1991:15:341-5.

6 Schluter B, Buschatz D, Trowitzsch E. Polysomnographic reference curves for the first and second year of life. Somnologie 2001:5:3-16.

7 Stebbens VA, Poets CF, Alexander JR, et al. Oxygen saturation and breathing patterns in infancy. 1: Full term infants in the second month of life. Arch Dis Child 1991:66:569-73

8 Thilo EH, Park-Moore B, Berman ER, et al. Oxygen saturation by pulse oximetry in healthy infants at an altitude of $1610 \mathrm{~m}(5280 \mathrm{ft})$ what is normal? Am J Dis Child 1991; 145:1137-40.

9 Levene S, McKenzie SA. Transcutanous oxygen saturation in sleeping infants: prone and supine. Arch Dis Child 1990;65:524-6.

10 Rhein L, Simoneau T, Davis J, et al. Reference values of nocturnal oxygenation for use in outpatient oxygen weaning protocols in premature infants. Pediatr Pulmonol 2011:47:453-9.

11 Urschitz MS, Wolff J, von Einem V, et al. Reference values for nocturnal home pulse oximetry during sleep in primary school children. Chest 2003;123:96-101.

12 Ellsbury DL, Acarregui MJ, McGuinness GA, et al. Controversy surrounding the use of home oxygen for premature infants with bronchopulmonary dysplasia. J Perinatol 2004; $24: 36-40$

13 MacLean JE, Fitzgerald DA. A rational approach to home oxygen use in infants and children. Paediatr Respir Rev 2006:7:215-22.

14 Fitzgerald DA, Massie RJ, Nixon GM, et al. Infants with chronic neonatal lung disease: recommendations for the use of home oxygen therapy. Med J Aust 2008;189:578-82.

15 Huppi PS. Oxygen saturation mon itoring for the preterm infant: the evidence basis for current practice. Pediatr Res 2009;65:375-80.

16 Nixon GM, Kermack AS, Davis GM, et al. Planning adenotonsillectomy in children with obstructive sleep apnea: the role of overnight oximetry. Pediatrics 2004;113:e19-25.

17 Harigopal S, Satish HP, Taktak AFG, et al. Oxygen saturation profile in healthy preterm infants. Arch Dis Child Fetal Neonatal Ed 2011;96:F339-42.

$18 \mathrm{Ng} \mathrm{A}$, Subhendar N, Primhak RA, et al. Arterial oxygen saturation profiles in healthy preterm infants. Arch Dis Child Fetal Neonatal Ed 1998;79:F64-6.

19 Nguyen CD, Dakin C, Yuill $M$, et al. The effect of sigh on cardiorespiratory synchronisation in healthy sleeping infants. Sleep 2012;35:1643-50.

20 Sankupellay M, Wilson S, Heussler HS, et al. Characteristics of sleep EEG power spectra in healthy infants in the first two years of life. Clin Neurophysiol 2010;122:236-43.

21 Terrill PI, Wilson SJ, Suresh S, et al. Application of recurrence quantification analysis to automatically estimate infant sleep states using a single channel of respiratory data. Med Biol Eng Comput 2012;50:851-65.

22 Terrill PI, Wilson SJ, Suresh $\mathrm{S}$, et al. Attractor structure discriminates sleep states: recurrence plot analysis applied to infant breathing patterns. IEEE Trans Biomed Eng 2010;57:1108-16.

23 Kuczmarski RJ, Ogden CL, Grummer-Strawn LM, et al. CDC growth charts: United States. Adv Data 2000:8(314):1-27.

24 Thoracic Society of Australia and New Zealand, Australasian Sleep Association. Accreditation of sleep disorders services, including standards for Paediatric Laboratories. 2000.

25 Anders TF. Manual of standardized terminology, techniques, and criteria for scoring of states of sleep and wakefulness in newborn infants (Mstni) eds-Anders, Emde, Parmelee. Psychophysiology 1972;9:97.

26 Iber C. The AASM manual for the scoring of sleep and associated events: rules, terminology and technical specifications. Westchester, IL: American Academy of Sleep Medicine, 2007

27 Grigg-Damberger M, Gozal D, Marcus CL, et al. The visual scoring of sleep and arousal in infants and children. J Clin Sleep Med 2007;3:201-43.

28 Anders $\mathrm{T}$, Emde $\mathrm{R}$, Parmalee $\mathrm{A}$, eds. A manual of standardized terminology techniques and criteria for scoring states of sleep and wakefulness in newborn infants. Los Angeles: UCLA brain information service, 1971

29 Iber C, Ancoli-Israel S, Chesson A, et al. The AASM manual for the scoring of sleep and associated events: rules, terminology and technical specification. American Academy of Sleep Medicine, 2007.

30 Thilo EH, Andersen D, Wasserstein ML, et al. Saturation by pulse oximetry: comparison of the results obtained by instruments of different brands. J Pediatr 1993;122:620-6.

31 Masters IB, Goes AM, Healy L, et al. Age related changes in oxygen saturation over the first year of life: a longitudinal study. J Paediatr Child Health 1994:30:423-8.

32 Meyts I, Van Reempts P, De Boeck K. Monitoring haemoglobin oxygen saturation in healthy infants using a new generaion pulse oximeter which takes motion atrifacts into account. Eur J Pediatr 2002;161:653-5. 